\title{
Apport du polymorphisme alloenzymatique à l'identification variétale de l'olivier (Olea europaea L)
}

\author{
N Ouazzani ${ }^{1 *}, \mathrm{R}$ Lumaret ${ }^{2}$, P Villemur ${ }^{3}$ \\ 1 École nationale d'agriculture, département d'arboriculture, BP S40, Meknès, Maroc; \\ 2 CEFE-CNRS, département de biologie des populations, BP 5051, F34033 Montpellier cedex 1; \\ 3 ENSA-INRA, laboratoire d'arboriculture, F34060 Montpellier cedex 1, France
}

(Reçu le 1er août 1994 ; accepté le 24 mars 1995)

\begin{abstract}
Résumé - L'identification des variétés d'olivier (Olea europaea $L$ ) est réalisée à partir du polymorphisme alloenzymatique des feuilles obtenu par électrophorèse sur gel d'amidon. L'analyse a porté sur 47 variétés représentant une large distribution de l'olivier dans le bassin méditerranéen. Vingt et un allèles ont été observés pour l'ensemble des 9 loci polymorphes étudiés ( 7 systèmes enzymatiques différents). Trente-huit génotypes multiloci ont permis l'identification de 35 variétés sur les 47 analysées. À partir de ces génotypes, les méthodes d'analyses multivariées ont abouti à classer les variétés en 6 groupes dont une minorité seulement représentait une région géographique précise (un groupe de 3 variétés françaises), 10 des 11 variétés originaires d'Afrique du Nord se retrouvant dans un même groupe. La composition très cosmopolite des autres groupes atteste de l'ampleur des diverses migrations humaines qui ont favorisé la dispersion de l'olivier sur l'ensemble du bassin méditerranéen. La combinaison de ces marqueurs enzymatiques avec des caractères morphologiques, physiologiques et agronomiques pourrait contribuer à la mise en place d'une classification systématique fiable des variétés d'olivier. La possibilité de l'utilisation des marqueurs alloenzymatiques pour l'amélioration variétale de l'olivier est également suggérée.
\end{abstract}

Olea europaea $L$ = olivier / polymorphisme alloenzymatique / identification variétale

Summary - Contribution of allozyme polymorphism to varietal identification in the olive tree (Olea europaea L). Allozyme polymorphism of leaf proteins was used to characterize 47 olive varieties widely distributed in the Mediterranean basin. Twenty-one alleles were observed at 9 polymorphic loci corresponding to 7 enzyme systems. Thirty-five of the 47 varieties could be identified by one of the 38 multiloci genotypes observed. Six groups of varieties were constituted using multivariate analysis of the multiloci genotypes. A single group gathered together varieties from a specific geographic area, namely France. Ten of the 11 varieties from North Africa were grouped together. Most of the groups contained varieties from various geographic origins, which was assumed to be the result of the occurrence of numerous human migrations which favoured olive dispersion throughout the Mediterranean basin. A combination of enzyme markers with morphological, physiological and agronomic characteristics may provide a consistent systematic classification of olive varieties. The possibility of using allozyme markers for olive breeding is also suggested.

Olea europaea $L=$ olive tree / allozyme polymorphism / varietal identification

* Correspondance et tirés à part 


\section{INTRODUCTION}

L'olivier (Olea europaea L), cultivé depuis l'Antiquité, compte de nombreuses variétés dont les plus anciennes résulteraient de croisements naturels et successifs entre les variétés ancestrales et/ou les formes spontanées ou sous-spontanées locales (Ciferri, 1950 ; Turrill, 1951 ; Zohary et Spiegel-Roy, 1975). En l'état actuel des recherches, les origines des variétés d'olivier cultivé restent imprécises et difficiles à élucider.

De nombreuses variétés identifiées ont fait l'objet de descriptions répondant à des objectifs agronomiques. Ruby (1918) base sa description sur les caractères du fruit, et Hauville (1953) s'appuie plutôt sur les caractéristiques du noyau. Baldini et Scaramuzzi (1963), et Barranco et Rallo (1984) définissent aussi un large assortiment variétal sur des bases morphologiques et agronomiques. Ces critères étudiés dans les collections de différents pays (Roselli et Scaramuzzi, 1974 ; Scaramuzzi et Roselli, 1986 ; Caballero et al, 1990) mettent en évidence une variabilité morphologique importante pour la taille du fruit et de la feuille ainsi que pour la teneur en huile. Par ailleurs, des études sur la biologie de l'espèce, son régime de reproduction (auto et inter-incompatibilité pollinique, richesse pollinique, stérilité mâle) soulignent la diversité rencontrée chez les variétés cultivées (Baldini et Guccione, 1952 ; Garieni-Gaddes, 1970 ; Morettini, 1972 ; Villemur et al, 1984).

De nombreuses synonymies de variétés locales inféodées souvent à une région donnée sont notées dans la majorité des pays oléicoles et il n'est pas rare de compter plus d'une centaine de variétés d'olivier dans chacun des principaux pays producteurs d'olive (Prevost et al, 1990 ; Tous et al, 1990 ; Rugini et Lavee, 1992).

Compte tenu de la richesse de ces informations et des nombreuses investigations effectuées chez les variétés de l'olivier, des méthodes basées sur des marqueurs stables, souvent à déterminisme monogénique ou oligogénique et en général sans valeur adaptative (De Vienne, 1984 ; Ohta, 1992), telles les protéines enzymatiques, sont susceptibles de présenter un intérêt certain pour une identification plus approfondie de ce matériel. L'électrophorèse d'isozymes, en permettant d'étudier les produits primaires de gènes de structure, est une technique particulièrement adaptée à l'identification variétale chez plusieurs espèces fruitières (Torres, 1989 ; Weeden, 1989). Cependant cette technique est soumise à certaines limites, notamment la difficulté de détecter certaines mutations qui n'affec- tent pas la charge de l'enzyme et la nécessité d'étudier un nombre élevé de marqueurs pour l'identification de clones ou de variétés très étroitement apparentés (Cheliak et Pitel, 1984 ; Chevreau, 1984).

Jusqu'à présent, chez l'olivier, les travaux utilisant l'électrophorèse enzymatique pour l'identification variétale ont concerné les phénotypes isozymiques du pollen (Pontikis et al, 1980 ; Trujillo et al, 1990). Ces phénotypes (sans interprétation génétique) basés sur l'intensité et le nombre de bandes observées ont mis en évidence une variation substantielle. Cependant, l'utilisation du pollen peut conduire à des confusions car un mélange de très nombreux grains de pollen est généralement utilisé pour l'extraction. Dans le cas d'isozymes spécifiées par plusieurs loci non identifiés, différentes combinaisons d'allèles pourraient être révélées à chaque échantillonnage (Torres, 1983). Dans le cas des enzymes dimères, le mélange de grains de pollen issus de la méiose provenant d'un individu hétérozygote peut produire seulement 2 homodimères. En outre, seuls les individus adultes ayant atteint la maturité de floraison peuvent être utilisés.

Pour ces raisons et pour parvenir à une identification fiable, nous avons choisi de travailler à partir d'extraits foliaires avec des systèmes enzymatiques dont le déterminisme génétique et les conditions d'expression ont été étudiés précédemment (Ouazzani et al, 1993). En outre, les feuilles ont l'intérêt d'être présentes pendant tous les stades de développement de l'arbre et sont aisées à prélever. Nous présentons ici les résultats d'utilisation du polymorphisme des allozymes des feuilles pour l'identification des variétés d'olivier.

\section{MATÉRIELS ET MÉTHODES}

\section{Matériel végétal}

Le matériel végétal utilisé dans cette étude est constitué de variétés provenant de la collection d'olivier de la station de recherches fruitières de l'Institut national de la recherche agronomique (INRA) de Montpellier et de prospections réalisées au Maroc. Les 47 variétés analysées (tableau I), les plus couramment utilisées, sont originaires des principaux pays producteurs et représentent une large distribution dans le bassin méditerranéen. Une partie de ces variétés a été analysée précédemment (Ouazzani et al, 1993). Un seul arbre par variété a été étudié, à l'exception des variétés Picholine (16 arbres), Picholine marocaine (4 arbres), Dahbia (4 arbres), et Grappola, Belgentier, Cailletier, et Verdale ( 2 arbres pour chaque variété). 


\section{Analyse enzymatique}

L'ensemble des variétés a été analysé par électrophorèse enzymatique sur gel d'amidon à partir d'extraits protéiques des feuilles. Les techniques d'extraction des protéines à partir des feuilles fraîches, de migration et de révélation des enzymes, et le déterminisme génétique des systèmes enzymatiques utilisés sont celles décrites par Ouazzani et al (1993). L'analyse a porté sur 9 loci polymorphes sur les 11 loci étudiés : des leucine aminopeptidases, E.C.3.4.11.1 (locus LAP1), des estérases, E.C.3.1.1.2 (loci EST1 et EST2), des alcool déshydrogénases, E.C.1.1.1.1 (locus ADH1), des malate déshydrogénases, E.C.1.1.1.37 (locus MDH2), des phosphoglucose isomérases, E.C.5.3.1.9 (PGl1-2, loci dupliqués présentant les mêmes allèles), des phosphoglucomutases, E.C.5.3.1.9 (locus PGM2), et des peroxidases, E.C.1.11.1.7 (locus PX1). Les loci PGM1 et MDH1, sont monomorphes dans nos conditions expérimentales.

\section{Analyse statistique}

Les génotypes décrits aux 9 loci étudiés ont été regroupés en génotype multiloci pour l'identification de chaque variété. La matrice de distance du $\chi^{2}$ pondérée (Benzecri, 1973) est utilisée pour la classification ascendante hiérarchique $(\mathrm{CAH})$ des variétés étudiées. Pour cette analyse, le génotype multiloci de chaque variété est codé selon la méthode décrite par Mathieu et al (1990) : on affecte les valeurs 0, 1 ou 2 respectivement si l'allèle est absent, présent à l'état hétérozygote ou présent à l'état homozygote. Pour les PGI (présence de gènes dupliqués présentant les mêmes allèles), on affecte la valeur 0 si l'allèle est absent, les valeurs 1,2 ou 3 respectivement s'il est présent 1,2 ou 3 fois et la valeur 4 si l'allèle est présent à l'état homozygote. Le critère d'agglomération utilisé est la distance «moyenne», qui a la propriété de ne pas déformer les distances initiales (Legendre et Legendre, 1979 ; Roux, 1985). Les groupements de génotypes sont déterminés pour un niveau d'agglomération correspondant à un pallier important dans l'organisation de la classification hiérarchique. Les analyses multivariées et la classification hiérarchique sont réalisées à l'aide du logiciel BIOMECO (Roux et Lebreton, 1988).

\section{RÉSULTATS}

\section{Polymorphisme enzymatique}

Chaque variété est identifiée par les génotypes obtenus pour les 9 loci polymorphes (tableau I). L'échantillon des variétés analysées présente en moyenne 2,77 allèles par locus avec une propor- tion moyenne de loci hétérozygotes par variété de $19,75 \%$ (minimum 0 , maximum $57,14 \%$, valeur calculée pour 7 loci, non compris les loci PGl1-2 pour lesquels le génotype double homozygote ne se distingue pas du génotype double hétérozygote). Vingt et un allèles ont été observés au total, dont 3 peuvent être considérés comme des allèles rares. C'est le cas des allèles EST11,07 (a), ADH10,96 (b) et $\mathrm{ADH}_{11,04}$ (c), observés une seule fois chez la variété Sofralik originaire de Turquie (présence des allèles $b$ et c), et Coratina originaire d'Italie (allèle a). Dans l'échantillon des variétés analysées, 6 génotypes différents ont été observés aux loci PGI1-2, 5 au locus LAP1 (les plus polymorphes), 3 aux loci EST1 et $A D H 1$, et 2 à chacun des loci EST2, MDH2, PGM2, et PX1 (tableau I).

\section{Identification variétale}

La discrimination entre les variétés analysées est basée sur leurs génotypes observés aux 9 loci étudiés (tableau I). Trente-huit génotypes multiloci différents ont été observés (sur les 4320 génotypes multiloci possibles) pour les 47 variétés analysées. Ces 38 génotypes ont permis l'identification de 35 variétés, ce qui donne un pourcentage d'indétermination de $25,53 \%$. Les autres variétés se répartissent entre les 3 génotypes restants : un génotype regroupe 8 variétés (Verdale $\left(n^{\circ} 23\right)$, Gaidourolia $\left(n^{\circ} 27\right)$, Belle d'Espagne ( $\left.n^{\circ} 29\right)$, Meslala $\left(n^{\circ} 36\right)$, Branquita $\left(n^{\circ} 38\right)$, Barouni $\left(n^{\circ} 40\right)$, Meski $\left(n^{\circ} 42\right)$, et Ayvalik $\left(n^{\circ} 44\right)$ ), et 2 génotypes regroupent chacun 2 variétés (Carydolia $\left(n^{\circ} 3\right)$ et Cornicabra $\left(n^{\circ} 7\right)$ d'une part et Salonenque $\left(n^{\circ} 21\right)$ et Dahbia $\left.\left(n^{\circ} 34\right)\right)$ d'autre part. Signalons que les individus analysés en répétition pour quelques variétés ont présenté le même génotype.

Pour la classification ascendante hiérarchique $(\mathrm{CAH})$, un génotype multiloci porteur de 2 variables observées une seule fois (allèles $A D H 10,96$ et $A D H 11^{1,04}$, variété Sofralik), est placé en élément supplémentaire pour qu'il n'influe pas sur l'analyse. La plupart des variétés forment des groupes à des niveaux d'agglomération inférieurs à $5 \%$ (fig 1). Au niveau $15 \%$ de la classification, 6 groupes de variétés sont mis en évidence.

Le groupe I rassemble 5 variétés caractérisées par le même génotype aux loci LAP1 (génotype «22»), ADH1 (génotype «22»), PGM2 (génotype «11"), et PX1 (génotype «22»). Le groupe II rassemble 27 variétés caractérisées par le même génotype aux loci LAP1 (génotype 
Tableau I. Génotypes multiloci a des 47 variétés d'olivier analysées pour 9 loci polymorphes.

\begin{tabular}{|c|c|c|c|c|c|c|c|c|c|c|}
\hline Vari & riétés & $\begin{array}{l}\text { Origine } \\
\text { géographique }\end{array}$ & LAP1 & EST1 & EST2 & $\begin{array}{l}\text { Locus } \\
A D H 1\end{array}$ & MDH2 & PGI1-2 & PGM2 & $P X 1$ \\
\hline 1 & Chemlal & Algérie & 22 & 12 & 22 & 22 & 22 & 1122 & 11 & 22 \\
\hline 2 & Sigoise & Algérie & 33 & 11 & 12 & 22 & 12 & 1122 & 11 & 22 \\
\hline 3 & Carydolia & Crète & 33 & 11 & 22 & 22 & 12 & 1122 & 11 & 22 \\
\hline 4 & Koroneiki & Crète & 13 & 11 & 12 & 22 & 22 & 1112 & 11 & 22 \\
\hline 5 & Mastoidis & Crète & 33 & 11 & 22 & 22 & 12 & 1122 & 12 & 22 \\
\hline 6 & Arbequine & Espagne & 33 & 12 & 22 & 12 & 12 & 1112 & 12 & 22 \\
\hline 7 & Cornicabra & Espagne & 33 & 11 & 22 & 22 & 12 & 1122 & 11 & 22 \\
\hline 8 & Hojiblanca & Espagne & 12 & 11 & 22 & 12 & 22 & 1112 & 11 & 22 \\
\hline 9 & Manzanille & Espagne & 33 & 11 & 22 & 12 & 12 & 1333 & 11 & 22 \\
\hline 10 & Picual & Espagne & 33 & 11 & 12 & 22 & 12 & 1112 & 12 & 22 \\
\hline 11 & Aglandeau & France & 33 & 11 & 22 & 22 & 12 & 4444 & 11 & 22 \\
\hline 12 & Amellau & France & 33 & 11 & 22 & 22 & 12 & 4444 & 11 & 11 \\
\hline 13 & Belgentier & France & 33 & 11 & 22 & 12 & 12 & 1112 & 12 & 22 \\
\hline 14 & Bouteillan & France & 33 & 11 & 22 & 22 & 22 & 1122 & 11 & 22 \\
\hline 15 & Cailletier & France & 33 & 11 & 12 & 22 & 22 & 1122 & 12 & 11 \\
\hline 16 & Cayon & France & 22 & 11 & 12 & 22 & 12 & 1122 & 11 & 22 \\
\hline 17 & Corniale & France & 33 & 11 & 22 & 22 & 22 & 4444 & 11 & 11 \\
\hline 18 & Lucques & France & 33 & 11 & 22 & 12 & 12 & 1222 & 11 & 11 \\
\hline 19 & Oliviere & France & 33 & 11 & 22 & 12 & 22 & 1112 & 11 & 22 \\
\hline 20 & Picholine & France & 33 & 12 & 22 & 22 & 12 & 1112 & 12 & 22 \\
\hline 21 & Salonenque & France & 33 & 11 & 22 & 22 & 12 & 1112 & 11 & 22 \\
\hline 22 & Tanche & France & 33 & 11 & 22 & 22 & 12 & 1122 & 11 & 11 \\
\hline 23 & Verdale & France & 33 & 11 & 22 & 22 & 22 & 1112 & 11 & 22 \\
\hline 24 & Amygdalolia & Grèce & 33 & 11 & 22 & 22 & 22 & 1222 & 12 & 22 \\
\hline 25 & Carolia & Grèce & 22 & 11 & 22 & 22 & 12 & 1111 & 11 & 22 \\
\hline 26 & Cothreiki & Grèce & 12 & 11 & 22 & 22 & 22 & 4444 & 11 & 22 \\
\hline 27 & Gaidourolia & Grèce & 33 & 11 & 22 & 22 & 22 & 1112 & 11 & 22 \\
\hline 28 & Ascolana & Italie & 13 & 11 & 22 & 12 & 12 & 1122 & 12 & 22 \\
\hline 29 & Belle d'Espagne & Italie & 33 & 11 & 22 & 22 & 22 & 1112 & 11 & 22 \\
\hline 30 & Coratina & Italie & 11 & 13 & 12 & 12 & 12 & 1122 & 11 & 22 \\
\hline 31 & Cypressino & Italie & 11 & 11 & 12 & 22 & 22 & 1111 & 11 & 22 \\
\hline 32 & Grappola & Italie & 12 & 11 & 22 & 12 & 22 & 1112 & 12 & 22 \\
\hline 33 & Bouchouika & Maroc & 33 & 11 & 12 & 22 & 22 & 1112 & 11 & 22 \\
\hline 34 & Dahbia & Maroc & 33 & 11 & 22 & 22 & 12 & 1112 & 11 & 22 \\
\hline 35 & Hamrani & Maroc & 33 & 11 & 12 & 12 & 12 & 1112 & 12 & 22 \\
\hline 36 & Mesiala & Maroc & 33 & 11 & 22 & 22 & 22 & 1112 & 11 & 22 \\
\hline 37 & Picholine M & Maroc & 33 & 11 & 12 & 22 & 12 & 1112 & 11 & 22 \\
\hline 38 & Branquita & Portugal & 33 & 11 & 22 & 22 & 22 & 1112 & 11 & 22 \\
\hline 39 & Sourani & Syrie & 22 & 11 & 22 & 22 & 12 & 1122 & 11 & 22 \\
\hline 40 & Barouni & Tunisie & 33 & 11 & 22 & 22 & 22 & 1112 & 11 & 22 \\
\hline 41 & Bidelhaman & Tunisie & 33 & 11 & 22 & 22 & 22 & 1122 & 12 & 22 \\
\hline 42 & Meski & Tunisie & 33 & 11 & 22 & 22 & 22 & 1112 & 11 & 22 \\
\hline 43 & PFR.5 & Tunisie & 33 & 11 & 22 & 22 & 22 & 1222 & 11 & 22 \\
\hline 44 & Ayvalik & Turquie & 33 & 11 & 22 & 22 & 22 & 1112 & 11 & 22 \\
\hline 45 & Domat & Turquie & 22 & 11 & 22 & 22 & 22 & 1333 & 11 & 22 \\
\hline 46 & Memecik & Turquie & 22 & 11 & 22 & 22 & 12 & 1112 & 11 & 22 \\
\hline 47 & Sofralik & Turquie & 33 & 11 & 22 & 34 & 22 & 1122 & 12 & 22 \\
\hline
\end{tabular}

a Mobilité relative des allèles aux loci : LAP1 : $1=0,92 ; 2=0,97 ; 3=1,00 ;$ EST1 $: 1=1,00 ; 2=1,04 ; 3=1,07 ;$ EST2 $: 1=0,94 ; 2=$ 1,$00 ;$ ADH1 $: 1=0,92 ; 2=1,00 ; 3=0,96 ; 4=1,04 ; \mathrm{MDH} 2: 1=0,79 ; 2=1,00 ; \mathrm{PGl}-2: 1=1,00 ; 2=1,60 ; 3=0,95 ; 4=1,05$; PGM2: $1=1,00 ; 2=1,14 ; P X 1: 11$ (présentant une activité) et 22 (sans activité). 


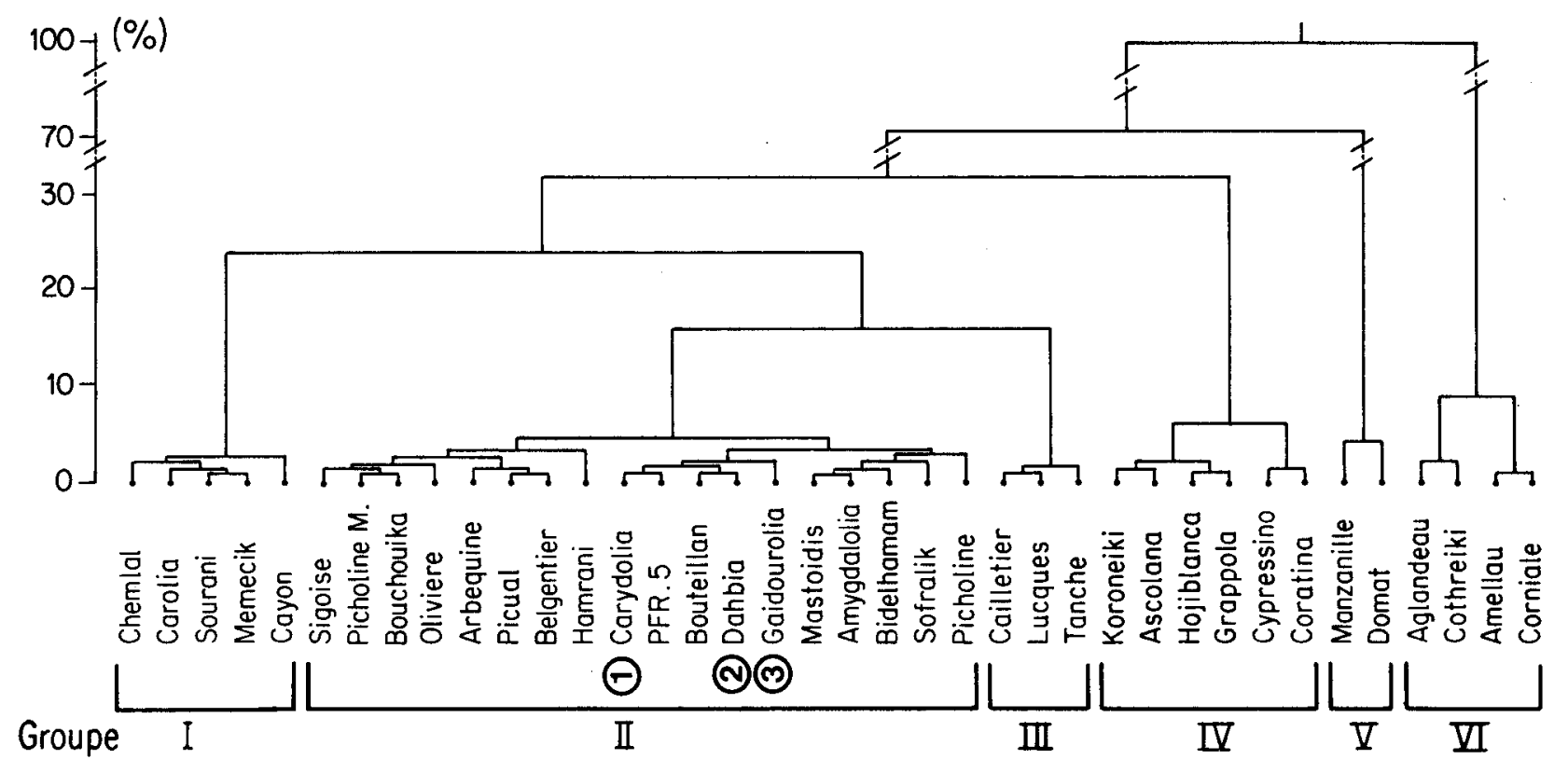

Fig 1. Relations entre les 47 variétés d'olivier étudiées à l'aide d'un dendrogramme issu de la classification hiérarchique ascendante réalisée sur la matrice de distance du $\chi^{2}$. Variétés présentant le même génotype multiloci : (1) Carydolia et Cornicabra ; (2) Dahbia et Salonenque ; (3) Gaidourolia et Belle d'Espagne, Branquita, Verdale, Meski, Barouni, Meslala et Ayvalik.

«33») et PX1 (génotype «22»). Le groupe III rassemble 3 variétés caractérisées par le même génotype aux loci LAP1 (génotype «33»), EST1 (génotype "11»), et PX1 (génotype "11»). Le groupe IV rassemble 6 variétés caractérisées par la présence de l'allèle LAP $1^{0,92}$ et le même génotype au locus PX1 (génotype «22»). Le groupe $V$ rassemble 2 variétés caractérisées par le même génotype aux loci PGl1-2 (génotype “1333»), EST1 (génotype "11»), EST2 (génotype «22»), PGM2 (génotype «11»), et PX1 (génotype «22»). Le groupe VI rassemble 4 variétés caractérisées par le même génotype aux loci PGI1-2 (génotype “4444»), EST1 (génotype «11»), EST2 (génotype «22»), ADH1 (génotype «22»), et PGM2 (génotype «11»). Au niveau $20 \%$ d'agglomération le groupe III rejoint le groupe II (fig 1).

Par ailleurs, on remarque que, dans la plupart des groupes, les variétés possèdent des origines géographiques diversifiées (tableau I et fig 1). Par exemple, le groupe I rassemble des variétés originaires d'Algérie, France, Grèce, Syrie, et Turquie. Seul le groupe III, composé uniquement de variétés originaire de France, fait exception. En outre, 10 des 11 variétés originaires d'Afrique du Nord se retrouvent dans le groupe II, et l'ensemble IV regroupe principalement les variétés originaires du centre de la Méditerranée (Italie et Crète, 5 variétés sur 6 ).

\section{DISCUSSION}

Le pourcentage de loci polymorphes (p) $(82 \%)$, le nombre moyen d'allèles par locus polymorphe (a) $(2,77)$ et la proportion moyenne de loci hétérozygotes par variété $(h)(19,75 \%)$ indiquent que le polymorphisme enzymatique de l'olivier cultivé est globalement similaire à celui d'autres espèces fruitières cultivées, telles le palmier dattier $(p=87 \% ; a=1,8 ;$ et $h=25,36 \%)$ et les agrumes $(p=64 \% ; a=2,8$; et $h=33,44 \%)$ (Torres et Tisserat, 1980 ; Torres, 1983). Ainsi, malgré une domestication intensive depuis l'Antiquité, l'olivier cultivé possède un polymorphisme important et des ressources génétiques encore exploitables, comme cela a été trouvé chez d'autres espèces d'arbres à longue durée de vie (Hamrick et al, 1979).

Pour déterminer la puissance des marqueurs enzymatiques, mis en évidence, pour l'identification des variétés d'olivier, nous avons calculé la probabilité pour laquelle 2 variétés différentes aient le même génotype multiloci, en considérant nos 47 variétés comme un échantillon représentatif de toutes les combinaisons génotypiques existantes. Pour cela, nous calculons les fréquences génotypiques pour chacun des 9 loci observés. Pour le locus LAP1 (tableau I), nous trouvons 2 (11), 6 (22), 34 (33), 3 (12), et 2 (13), soit, en fréquence, $0,04,0,13,0,72,0,07$ et 0,04 . 
La probabilité de tirer de cet ensemble 2 combinaisons ayant le génotype 11 est $(0,04)^{2}$ et la probabilité de tirer 2 combinaisons ayant le même génotype pour locus LAP1 est $(0,04)^{2}+$ $(0,13)^{2}+(0,72)^{2}+(0,07)^{2}+(0,04)^{2}=0,54$.

Le même calcul donne $0,85,0,67,0,64,0,50$, $0,33,0,62,0,80$ pour les loci EST1, EST2, ADH1, $\mathrm{MDH} 2$, PGI1-2 (considérés comme un seul locus), PGM2 et PX1, respectivement. Si les 8 loci sont indépendants (nous avons vérifié qu'ils le sont, au moins pour plusieurs d'entre eux (Ouazzani et al, 1993)), la probabilité recherchée est le produit des probabilités calculées par locus, soit $1,6 \%$. Pour les 47 variétés, nous devons nous attendre à voir 1 cas ou 2 pour lesquels 2 variétés auront le même génotypes multiloci. Dans nos résultats, nous avons observé 3 cas où un génotype multiloci correspond à 2 variétés ou plus. L'adjonction de marqueurs polymorphes supplémentaires pourrait conduire à une discrimination des variétés non identifiées. Ainsi, le polymorphisme enzymatique montre un potentiel d'identification variétale intéressant dans l'échantillon analysé. En effet, les 38 génotypes multiloci mis en évidence ont permis l'identification d'environ 3 quarts des variétés analysées, avec une discrimination plus importante pour les systèmes enzymatiques PGI, MDH, LAP. Ces même systèmes, dans le cas du pollen, ont montré une variation importante des phénotypes basée sur l'intensité et le nombre des bandes observées (Pontikis et al, 1980 ; Trujillo et al, 1990). De même, la discrimination entre les variétés originaires de Grèce (tableau I) confirme la variation observée dans le cas d'utilisation du pollen par Pontikis et al (1980).

On peut remarquer que la majorité des variétés identifiées diffère par l'identité des allèles, seulement à 1 ou 2 loci (tableau I). Ce résultat laisse supposer que la plupart des variétés d'olivier, notamment les plus anciennes, auraient une origine commune et présenteraient, quelle que soit l'origine géographique de la variété, une forte proportion d'ancêtres communs.

Peu de relations ont été observées entre les génotypes multiloci et l'origine géographique des variétés. Ce résultat peut s'expliquer par les diverses migrations humaines qui ont favorisé la dispersion de l'olivier cultivé depuis la plus haute Antiquité sur l'ensemble du pourtour de la Méditerranée (Chevalier, 1948 ; Ciferri, 1950). Par ailleurs, la présence de la plupart des variétés originaires d'Afrique du Nord dans un même groupe (groupe II, fig 1) ainsi que la ressemblance génotypique entre certaines de ces variétés (variétés Sigoise, Picholine marocaine, et
Bouchouika, tableau I et fig 1), laissent supposer une différenciation commune et originale d'une partie des variétés de cette région comme l'on suggéré Chevalier (1948), Ciferri (1950), et Hauville (1953).

Dans le cadre de cette étude, l'utilisation des marqueurs enzymatiques a permis de lever quelques ambiguïtés concernant l'identification de certaines variétés. Ainsi, on a pu lever l'équivoque sur la similitude entre les variétés "Picholine marocaine" $\left(n^{\circ} 37\right)$ et "Sigoise» $\left(n^{\circ} 2\right.$, originaire d'Algérie), puisque les génotypes multiloci des 2 variétés sont différents (tableau I). Cependant, la ressemblance des génotypes laisse supposer que ces 2 variétés sont étroitement apparentées (fig 1). En outre, elles présentent un génotype différent de celui de la variété Picholine originaire de la France. L'analyse d'un plus grand nombre d'individus, sous une même dénomination variétale, associés à des marqueurs polymorphes supplémentaires devrait apporter quelques éclaircissements concernant les nombreuses synonymies notées dans la majorité des pays oléicoles.

Les premières investigations dans l'identification des variétés d'olivier par les allozymes des feuilles sont encourageantes, comme cela a été trouvé chez d'autres arbres fruitiers reproduits par voie végétative (Valdeyron et Valizadeh, 1976, cas du figuier). La combinaison de ces marqueurs génétiques avec des caractères morphologiques et biologiques pourrait contribuer à la mise en place d'une classification systématique fiable des variétés d'olivier.

Les regroupements de variétés mis en évidence par les génotypes multiloci ne concordent pas, le plus souvent, avec les caractéristiques morphologiques et agronomiques connues chez les variétés analysées. Cependant, les marqueurs enzymatiques constituent un outil précieux d'étude de variabilité afin d'établir des programmes d'amélioration de l'olivier. Ces marqueurs devraient permettre, par exemple, à un stade précoce, de contrôler l'origine de la descendance, de détecter le pollen illégitime lors des croisements contrôlés, ainsi que d'orienter le choix des croisements, en exploitant l'hétérozygotie observée dans le but d'augmenter la variabilité.

\section{RÉFÉRENCES}

Baldini E, Guccione G (1952) Osservazioni di una razza di olivo con antere sterili. Ann Sperim Agr 6, $1-12$ 
Baldini E, Scaramuzzi F (1963) Le cultivar. In : Olive da Tavola, Edagricole, Bologna, 61-111

Barranco D, Rallo L (1984) Las variedades de olivo cultivadas en Andalucia. Minestario de Agricultura, Madrid

Benzecri JP (1973) L'analyse des données. II. L'analyse des correspondances. Dunod, Paris

Caballero JM, Del Rio C, Eguren J (1990) Further agronomical information about a world collection of olive cultivars. Acta Hort 286, 45-48

Cheliak WM, Pitel JA (1984) Electrophoretic identification of clones in trembling aspen. Can $J$ for Res 14 , 740-743

Chevalier A (1948) L'origine de l'olivier cultivé et ses variations. Rev Int Bot Appl Agric Trop 28, 1-25

Chevreau E (1984) Contribution à l'étude de la génétique du pommier : apport de l'analyse enzymatique. Thèse, Orsay, Paris

Ciferri R (1950) Éléments pour l'étude de l'origine et de l'évolution de l'olivier cultivé. Acte XIII Congrès international d'oléiculture 1, 189-194

De Vienne D (1984) Limites et perspectives des marqueurs moléculaires. Le Sélectionneur Français 33, $35-46$

Garieni-Gaddes RM (1970) Qualité des fleurs et pollinisation croisée de l'olivier en Tunisie. $3^{e}$ Conférence internationale sur les techniques oléicoles, Torremolinos, Espagne, 1-6

Hamrick JL, Linhart YB, Mitton JB (1979) Relationships between life history characteristics and electrophoretically detectable genetic variation in plants. Annu Rev Ecol Syst 10, 173-200

Hauville A (1953) La répartition des variétés d'oliviers en Algérie et ses conséquences pratiques. Bull Soc Agric Algérie 580, 1-8

Legendre L, Legendre P (1979) Écologie numérique. II. La structure des données écologiques. Masson, Paris

Mathieu E, Autem M, Roux M, Bonhomme F (1990) Épreuves de validation dans l'analyse de structures génétiques multivariées. Rev Stat App/ 38, 47-66

Morettini A (1972) L'olivicoltura, 2nd ed, Ramo Editoriale degli Agricoltori, Roma

Ohta T (1992) The nearly neutral theory of molecular evolution. Annu Rev Ecol Syst 23, 263-286

Ouazzani N, Lumaret R, Villemur P, Di Giusto F (1993) Leaf allozyme variation in cultivated and wild olive trees (Olea europaea L). J Hered 84, 34-42

Pontikis CA, Loukas M, Kousounis G (1980) The use of biochemical markers to distinguish olive cultivars. J Hort Sci 55, 333-343
Prevost G, Bartolini G, Messeri C (1993) Cultivar italiane di olivo e loro sinonimi. Lucca

Roselli G, Scaramuzzi F (1974) Definizione dei termini di cultivar e clone. Atti Accademia Agricoltura Bologna 2, 119

Roux M (1985) Algorithmes de classification. Méthodes et programmes. Masson, Paris

Roux M, Lebreton JM (1988) Progiciel BIOMECO. Groupe de Biométrie CEFE-CNRS, Montpellier

Ruby J (1918) Recherches morphologiques et biologiques sur l'olivier et sur les variétés cultivées en France. Thèse, faculté des sciences, Paris

Rugini E, Lavee S (1992) Olive. In : Biotechnology of Perennial Fruit Crops (FA Hammerschlag, RE Litz, eds), Cambridge University Press, Cambridge, 371382

Scaramuzzi F, Roselli G (1986) Olive genetic improvement. Olea 17, 1-17

Torres AM (1983) Fruit trees. In : Isozymes in Plant Genetics and Breeding, part B (SD Tanksley, TJ Orton, eds), Elsevier, Amsterdam, 401-421

Torres AM (1989) Isozyme analysis of tree fruits. In : Isozymes in Plant Biology (DE Soltis, PS Soltis, eds), Dioscorides Press, Portland, OR, USA, 192205

Torres AM, Tisserat B (1980) Leaf isozymes as genetic markers in date palms. Amer J Bot 67, 162-167

Tous J, Romero A, Barronco D (1990) Olive cultivars in Catalonia (Spain). Acta Hort, 286, 129-132

Trujillo I, Rallo L, Carbonell EA, Asins MJ (1990) Isoenzymatic variability of olive cultivars according to their origin. Acta Hort 286, 137-140

Turrill WB (1951) Wild and cultivated olives. Kew Bull 3, 439-442

Valdeyron G, Valizadeh M (1976) L'identification variétale du figuier, Ficus carica L, par l'étude du polymorphisme enzymatique par électrophorèse. $C R$ Aca Agric 62, 170-175

Villemur P, Musho US, Delmas JM, Maamar M, Ouksili A (1984) Contribution à l'étude de la biologie florale de l'olivier (Olea europaea L) : stérilité mâle, flux pollinique et période effective de pollinisation. Fruits 39, 467-473

Weeden NF (1989) Applications of isozymes in plant breeding. In : Plant Breeding Reviews (J Janick, ed), Timber Press, Portland, OR, USA, 11-54

Wilson AC, Carlson SS, White IJ (1977) Biochemical evolution. Ann Rev Biochem 46, 573-639

Zohary D, Spiegel-Roy P (1975) Beginnings of fruit growing in the Old World. Science 187, 319-327 